\title{
Lattice Points in a Sphere
}

\section{Bleicher and M. I. Knopp i}

(March 26. 1964)

Let $R_{3}(x)$ be the remainder in the classical lattice point problem for a 3 -sphere of radius $\sqrt{x}$ and center $(0,0,0)$. We prove that as $x \rightarrow+\infty$,

$$
R_{3}(x)=O\left(x^{3 / 4} \log x\right)
$$

and

$$
R_{3}(x)=\Omega\left(x^{1 / 2} \log \log x\right) .
$$

Key Words: Additive analytic number theory, lattice points, remainder formulas.

\section{Introduction}

In this paper we consider the classical lattice point problem for the three-dimensional sphere. The problem can be described as follows. Let $x$ be a positive real number and let $k$ be a positive integer. Consider a $k$-dimensional sphere of radius $\sqrt{x}$ and center $(0, \ldots, 0)$. Following the notation of $\mathrm{W}$ alfisz [4], ${ }^{2}$ we let $A_{k}(x)$ be the number of integer lattice points in this sphere. A simple geometric argument shows that as $x \rightarrow+\infty, A_{k}(x) \sim V_{k}(x)$, where $V_{k}(x)$ is the volume of the sphere in question. The problem then is to get an asymptotic estimate of the difference $R_{k}(x)=A_{k}(x)-V_{k}(x)$.

Here we are considering only $R_{3}(x)=A_{3}(x)-\frac{4}{3} \pi x^{3 / 2}$. We obtain the following results:

$$
\begin{aligned}
& R_{3}(x)=O\left(x^{3 / 4} \log x\right), x \rightarrow+\infty \\
& R_{3}(x)=\Omega\left(x^{1 / 2} \log \log x\right), x \rightarrow+\infty .
\end{aligned}
$$

Of course (1) is not new. Vinogradov [3] has in fact shown that $R_{3}(x)=O\left(x^{\frac{19}{28}+\epsilon}\right), \epsilon>0$, an upper estimate better than (1). However this result depends upon his difficult theory of exponential sums. Our estimate (1), on the other hand, is better than the elementary result $A_{3}(x)=O(x)$ and depends only upon a fairly standard application of the circle method.

As far as we can ascertain (2) is new. It is based upon the $\Omega$-estimate for $R_{4}(x)$ [4, p. 95]

$$
R_{4}(x)=\Omega(x \log \log x), x \rightarrow+\infty .
$$

Walfisz [4, p. 94] gives only $R_{3}(x)=\Omega\left(x^{1 / 2}\right), x \rightarrow+\infty$. In [1] it is shown that $\lim _{x \rightarrow \infty}\left|R_{3}(x) x^{-1 / 2}\right|=+\infty$, but this is of course weaker than (3).

\section{Preliminaries}

Landau's formula for $A_{k}(x) \cdot(k \geqslant 4)$ is $[4$, p. 29]

$$
A_{k}(x)=\frac{\pi^{k / 2}}{\Gamma\left(\frac{k}{2}\right)} \sum_{1 \leqslant q \leqslant x^{1 / 2}} \sum_{h(\bmod q)}^{\prime}\left(\frac{S(h, q)}{q}\right)^{k} \cdot \sum_{1 \leqslant n \leqslant x} n^{k / 2-1} e^{-2 \pi i n h / q}+O\left(x^{k / 4} \log x\right), x \rightarrow+\infty .
$$

${ }^{1}$ Present address: The University of Wisconsin. Madison, Wis.

${ }^{2}$ Figures in brackets indicate the literature references at the end of this paper. 
Here $S(h, q)=\sum_{a(\bmod q)} e^{2 \pi i h a^{2} / q}$ is the famous Gaussian sum about which we need only the fact that

$$
|S(h, q)| \leqslant K q^{1 / 2}
$$

where $K$ is independent of $h$ and $q$ [4, p. 10]. The notation $\Sigma^{\prime}$ indicates that we are to sum over only those $h$ such that $(h, q)=1$.

If (4) held for $k=3$ we could apply it to derive (1) without much difficulty. However since the proof of (4) given in [4] fails for $k<4$, we replace it for $k=3$ with the following formula obtainable by the same general method.

$$
A_{3}(x)=2 \pi \sum_{n \leq x} n^{1 / 2}+O\left(x^{3 / 4} \log x\right), x \rightarrow+\infty .
$$

Once we have (6), (1) is easily obtainable.

We will also need the following standard result [4, p. 25].

Lemma $l$ (Euler Summation Formula). Let $\Psi(\mathrm{t})=\mathrm{t}-[\mathrm{t}]-\frac{1}{2} . \quad$ If $\mathrm{f}(\mathrm{t})$ has a continuous derivative in the interval $\mathrm{a} \leqslant \mathrm{t} \leqslant \mathrm{b}(\mathrm{a}<\mathrm{b})$, then

$$
\sum_{a<m \leqslant b} f(m)=\int_{a}^{b} f(t) d t+\Psi(a) f(a)-\Psi(b) f(b)+\int_{a}^{b} \Psi(t) f^{\prime}(t) d t .
$$

This is proved by integrating $\int_{a}^{b} \Psi(t) f^{\prime}(t) d t$ by parts.

\section{Proof of (6) and (1)}

Many of the calculations done in the proof of (4) [4, pp. 29-35] are valid for $k=3$. In particular we have $[4$, p. 33, formula (21)]

$$
\begin{aligned}
A_{3}(x)= & \sum_{q \leq x 1 / 2} \sum_{h(\bmod q)}^{\prime}\left(\frac{S(h, q)}{q}\right)^{3} \int_{\theta(h, q)} w^{-3 / 2} \\
& \sum_{n \leqslant x} \exp \left\{\frac{\pi n}{x}-2 \pi i n\left(y+\frac{h}{q}\right)\right\} d y+O\left(x^{3 / 4} \log x\right), x \rightarrow+\infty .
\end{aligned}
$$

In (8), $w=x^{-1}-2 y i$, and $\theta(h, q)$ is an interval described as follows. Let $h^{\prime} / q^{\prime}$ and $h^{\prime \prime} / q^{\prime \prime}$ be the two Farey fractions of order $x^{1 / 2}$ closest to $h / q$ with say $h^{\prime} / q^{\prime}<h / q<h^{\prime \prime} / q^{\prime \prime}$, and consider the interval $\left[\frac{h^{\prime}+h}{q^{\prime}+q}, \frac{h+h^{\prime \prime}}{q+q^{\prime \prime}}\right]$. Then $\theta(h, q)$ is obtained from this interval by translating $h / q$ to the origin, that is,

$$
\theta(h, q)=\left[\frac{h^{\prime}+h}{q^{\prime}+q}-\frac{h}{q}, \frac{h+h^{\prime \prime}}{q+q^{\prime \prime}}-\frac{h}{q}\right]
$$

For our purpose here the essential fact about $\theta(h, q)$ is [4, p. 30]

$$
\left\{\begin{array}{l}
|y| \leqslant q^{-1} x^{-1 / 2}, \text { for } y \epsilon \theta(h, q) \\
|y| \geqslant 2^{-1} q^{-1} x^{-1 / 2}, \text { for } y \notin \theta(h, q) .
\end{array}\right.
$$

for any Farey fraction $h / q$ or order $x^{1 / 2}$. 
By (8) we have

$$
\begin{aligned}
& A_{3}(x)=\int_{\theta(0,1)} w^{-3 / 2} \sum_{n \leq x} \exp \left\{\frac{\pi n}{x}-2 \pi i n y\right\} d y \\
&+\sum_{2 \leqslant q \leqslant x 1 / 2} \sum_{h(\bmod q)}^{\prime}\left(\frac{S(h, q)}{q}\right)^{3} \int_{\theta(h, q)} w^{-3 / 2} \sum_{n \leq x} \exp \left\{\frac{\pi n}{x}-2 \pi i n\left(y+\frac{h}{q}\right)\right\} d y \\
& \quad+O\left(x^{3 / 4} \log x\right), x \rightarrow+\infty .
\end{aligned}
$$

Again we observe that the calculations of [4, pp. 33-34] are valid for $k=3$. These yield

$$
\int_{\theta(0,1)} w^{-3 / 2} \sum_{n \leq x} \exp \left\{\frac{\pi n}{x}-2 \pi i n y\right\} d y=\int_{-\infty}^{\infty} w^{-3 / 2} \sum_{n \leq x} \exp \left\{\frac{\pi n}{x}-2 \pi i n y\right\} d y+O\left(x^{3 / 4}\right), x \rightarrow+\infty .
$$

Now

$$
\int_{-\infty}^{\infty} w^{-3 / 2} \sum_{n \leq x} \exp \left\{\frac{\pi n}{x}-2 \pi i n y\right\} d y=\sum_{n \leq x} e^{\pi n / x} \int_{-\infty}^{\infty} w^{-3 / 2} e^{-2 \pi i n y} d y
$$

and by [4, p. 35] (again valid for $k=3$ ),

$$
\int_{-\infty}^{\infty} w^{-3 / 2} e^{-2 \pi i n y} d y=\frac{\pi^{3 / 2}}{\Gamma(3 / 2)} e^{-\pi n / x_{n}^{1 / 2}}=2 \pi e^{-\pi n / x_{n}^{1 / 2}} .
$$

Thus, we have

$$
\int_{-\infty}^{\infty} w^{-3 / 2} \sum_{n \leq x} \exp \left\{\frac{\pi n}{x}-2 \pi i n y\right\} d y=2 \pi \sum_{n \leq x} n^{1 / 2}
$$

and (10) becomes

$$
\begin{aligned}
A_{3}(x)=2 \pi \sum_{n \leq x} n^{1 / 2}+ & \sum_{2 \leq q \leq x 1 / 2} \sum_{h(\bmod q)}^{\prime}\left(\frac{S(h, q)}{q}\right)^{3} \\
& \int_{\theta(h, q)} w^{-3 / 2} \sum_{n \leq x} \exp \left\{\frac{\pi n}{x}-2 \pi i n\left(y+\frac{h}{q}\right)\right\} d y+O\left(x^{3 / 4} \log x\right), x \rightarrow+\infty .
\end{aligned}
$$

Let $\Sigma$ denote the multiple sum on the right-hand side of (11); to prove (6) it is sufficient to show that $\Sigma=O\left(x^{3 / 4} \log x\right)$, as $x \rightarrow+\infty$.

By (5) and (9),

$$
|\Sigma| \leqslant K \sum_{2 \leqslant q \leqslant x 1 / 2} q^{-3 / 2} \sum_{h(\bmod q)}^{\prime} \int_{|y| \leqslant q^{-1} x^{-1 / 2}}|w|^{-3 / 2}\left|\sum_{n \leqslant x} \exp \left\{\frac{\pi n}{x}-2 \pi i n\left(y+\frac{h}{q}\right)\right\}\right| d y .
$$

We apply the familiar method of partial summation to estimate the inner sum. Let $T(n)$

$=\sum_{1 \leqslant k \leqslant n} e^{-2 \pi i k(y+h / q)}$. Then since $T(n)$ is a geometric series

$$
|T(n)| \leqslant 2\left|e^{\pi i(y+h / q)}-e^{-\pi i(y+h / q)}\right|-1=\left|\sin \pi\left(y+\frac{h}{q}\right)\right|^{-1} .
$$

Since $|y| \leqslant q^{-1} x^{-1 / 2}, q^{-1}\left(h-x^{-1 / 2}\right) \leqslant y+\frac{h}{q} \leqslant q^{-1}\left(h+x^{-1 / 2}\right)$, while $q \geqslant 2$ implies that $1 \leqslant h \leqslant q-1$; 
thus if $x \geqslant 1$ (say), $O \leqslant y+\frac{h}{q} \leqslant 1$. Therefore

$$
\left|\sin \pi\left(y+\frac{h}{q}\right)\right|^{-1} \leqslant \max \left\{\frac{1}{2(y+h / q)}, \frac{1}{2(1-y-h / q)}\right\}
$$

Also,

$q y+h \geqslant h-x^{-1 / 2} \geqslant h-\frac{1}{2}$, and $q-q y-h \geqslant q-h-x^{-1 / 2} \geqslant q-h-\frac{1}{2}$, if $x \geqslant 4$. We conclude that

$$
|T(n)| \leqslant q\left\{\frac{1}{2 h-1}+\frac{1}{2 q-2 h-1}\right\} \leqslant q\left\{\frac{1}{h}+\frac{1}{q-h}\right\} .
$$

Now,

$$
\begin{aligned}
\sum_{1 \leqslant n \leqslant x} \exp \left\{\frac{\pi n}{x}-2 \pi i n\left(y+\frac{h}{q}\right)\right\}=\sum_{1 \leqslant n \leqslant x} e^{\pi n / x}\{T(n)-T(n-1)\} & \\
& =\sum_{1 \leqslant n \leqslant x} T(n)\left\{e^{\pi n / x}-e^{\pi(n+1) / x}\right\}+e^{\pi(|x|+1) / x} T(|x|)
\end{aligned}
$$

and we have

$$
\begin{aligned}
\left|\sum_{1 \leqslant n \leqslant x} \exp \left\{\frac{\pi n}{x}-2 \pi i n\left(y+\frac{h}{q}\right)\right\}\right| & \leqslant q\left\{\frac{1}{h}+\frac{1}{q-h}\right\} \sum_{1 \leqslant n \leqslant x}\left\{e^{\pi(n+1) / x}-e^{\pi n / x}\right\} \\
+ & q\left\{\frac{1}{h}+\frac{1}{q-h}\right\} e^{\pi(|x|+1) / x} \leqslant 2 q\left\{\frac{1}{h}+\frac{1}{q-h}\right\} e^{\pi(x+1) / x} \leqslant K^{\prime} q \cdot\left\{\frac{1}{h}+\frac{1}{q-h}\right\} .
\end{aligned}
$$

where $K^{\prime}$ is independent of $h, q$, and $x$. This, with (12), leads to

$$
\sum=O\left(\sum_{2 \leqslant q \leqslant x^{1 / 2}} q^{-1 / 2} \sum_{h(\bmod q)}^{\prime}\left\{\frac{1}{h}+\frac{1}{q-h}\right\} \int_{0}^{q^{-1} x^{-1 / 2}}|w|^{-3 / 2} d y\right), x \rightarrow+\infty .
$$

But $|w|^{-3 / 2}=x^{3 / 2}\left(1+4 x^{2} y^{2}\right)^{-3 / 4} \leqslant \min \left\{x^{-3 / 2},(2 y)^{-3 / 2}\right\}$, so that

$$
\begin{aligned}
\sum & =O\left(\sum_{2 \leq q \leq x^{1 / 2}} q^{-1 / 2} \sum_{h(\bmod q)}^{\prime}\left\{\frac{1}{h}+\frac{1}{q-h}\right\}\left\{\int_{0}^{x^{-1}} x^{3 / 2} d y+\int_{x^{-1}}^{q^{-1} x^{-1 / 2}} y^{-3 / 2} d y\right\}\right) \\
& =O\left(\sum_{2 \leq \eta \leq x^{1 / 2}} q^{-1 / 2} \sum_{h(\bmod q)}^{\prime}\left\{\frac{1}{h}+\frac{1}{q-h}\right\} x^{1 / 2}\right) \\
& =O\left(x^{1 / 2} \sum_{2 \leq \eta \leq x^{1 / 2}} q^{-1 / 2} \log q\right) \\
& =O\left(x^{3 / 4} \log x\right), \text { as } x \rightarrow+\infty,
\end{aligned}
$$

and (6) is proved.

Tó obtain (1) we simply apply (7) to $\sum_{1 \leq n \leq x} n^{1 / 2}$. This gives

$$
\sum_{1 \leq n \leq x} n^{1 / 2}=\int_{0}^{x} t^{1 / 2} d t-\Psi(x) x^{1 / 2}+\frac{1}{2} \int_{0}^{x} \Psi(t) \cdot t^{-1 / 2} d t=\frac{2}{3} x^{3 / 2}+O\left(x^{1 / 2}\right), x \rightarrow+\infty .
$$


Together with (6), this implies

$$
A_{3}(x)=\frac{4}{3} \pi x^{3 / 2}+O\left(x^{3 / 4} \log x\right), x \rightarrow+\infty,
$$

and the proof of (1) is complete.

\section{Proof (2)}

We begin with two lemmas (cf. [4, pp. 49-50]).

LeMmA 2. $A_{k}(x)=\sum_{-\sqrt{x} \leqslant m \leqslant \sqrt{x}} A_{k-1}\left(x-m^{2}\right)$, for $k \geqslant 2$.

Proof: Clear.

LEMMA 3. $\sum_{-\sqrt{\mathrm{x}} \leq \mathrm{m} \leqslant \sqrt{\mathrm{x}}}\left(\mathrm{x}-\mathrm{m}^{2}\right)^{\mathrm{k} / 2}=\int_{-\sqrt{\mathrm{x}}}^{1 \overline{\mathrm{x}}}\left(\mathrm{x}-\mathrm{t}^{2}\right)^{\mathrm{k} / 2} \mathrm{dt}+\mathrm{O}\left(\mathrm{x}^{\frac{k-1}{2}}\right), \mathrm{x} \rightarrow+\infty$.

Proof: By Lemma 1,

$$
\sum_{-\sqrt{x} \leqslant m \leqslant \sqrt{x}}\left(x-m^{2}\right)^{k / 2}=\sum_{-\sqrt{x}<m \leqslant \sqrt{x}}\left(x-m^{2}\right)^{k / 2}=\int_{-\sqrt{\bar{x}}}^{\backslash \bar{x}}\left(x-t^{2}\right)^{k / 2} d t-k \int_{-\sqrt{\bar{x}}}^{\backslash \bar{x}} \Psi(t)\left(x-t^{2}\right)^{\frac{k}{2}-1} t d t .
$$

But by the second mean value theorem of the integral calculus,

$$
\int_{-\sqrt{x}}^{\backslash \bar{x}} \Psi(t)\left(x-t^{2}\right)^{\frac{k}{2}-1} t d t=O\left(x^{\frac{k}{2}-1+\frac{1}{2}}\right)=O\left(x^{\frac{k-1}{2}}\right) \text {, as } x \rightarrow+\infty,
$$

since $\int_{-\sqrt{x}}^{1 \bar{x}} \Psi(t) d t$ is bounded, independently of $x$.

To prove (2) we assume

$$
R_{3}(x)=o\left(x^{1 / 2} \log \log x\right), x \rightarrow+\infty
$$

and show that this leads to a contradiction. By lemma 2 , and the definition of $R_{3}(x)$,

$$
A_{4}(x)=\sum_{-\sqrt{x} \leqslant m \leqslant \sqrt{x}} A_{3}\left(x-m^{2}\right)=\frac{4}{3} \pi \sum_{-\sqrt{x} \leqslant m \leqslant \sqrt{x}}\left(x-m^{2}\right)^{3 / 2}+\sum_{-\sqrt{x} \leqslant m \leqslant \sqrt{x}} R_{3}\left(x-m^{2}\right) .
$$

By (13), given any $\epsilon>0$ there exists $N>3$ such that if $x>N$, then $\left|R_{3}(x)\right|<\epsilon x^{1 / 2} \log \log x$. Also (13) implies that for any $x>3,\left|R_{3}(x)\right|<K x^{1 / 2} \log \log x$, where $K$ is independent of $x$. Therefore, assuming that $x>N$, we have

$$
\begin{aligned}
\sum_{-\sqrt{x} \leqslant m \leqslant \sqrt{x}} R_{3}\left(x-m^{2}\right) \mid \leqslant & \sum_{-\sqrt{x-N<m<\sqrt{x-N}}}\left|R_{3}\left(x-m^{2}\right)\right|+\sum_{\sqrt{x}-N \leqslant|m| \leqslant \sqrt{x}}\left|R_{3}\left(x-m^{2}\right)\right| \\
<2 \epsilon(x-N)^{1 / 2} x^{1 / 2} \log \log x+\frac{K N}{(x-N)^{1 / 2}} x^{1 / 2} \log \log x & \\
& +R_{3}(0)+R_{3}(1)+R_{3}(2),
\end{aligned} .
$$


where we have used the fact that $x^{1 / 2} \log \log x$ is monotone and observed that there are at most $N /(x-N)^{1 / 2}$ integers in the range $\sqrt{x-N} \leqslant|m| \leqslant \sqrt{x}$. Now holding $N$ fixed and letting $x \rightarrow+\infty$, we have

$$
\varlimsup_{x \rightarrow+\infty} \frac{\left|\sum_{-\sqrt{x}<m<\sqrt{x}} R_{3}\left(x-m^{2}\right)\right|}{x \log \log x} \leqslant 2 \epsilon .
$$

Since $\epsilon>0$ is arbitrary, we conclude that

$$
\sum_{-\sqrt{x} \leqslant m \leqslant \sqrt{x}} R_{3}\left(x-m^{2}\right)=o(x \log \log x), \text { as } x \rightarrow+\infty,
$$

so that

$$
A_{4}(x)=\frac{4}{3} \pi \sum_{-\sqrt{\bar{x}} \leqslant m \leqslant \sqrt{x}}\left(x-m^{2}\right)^{3 / 2}+o(x \log \log x), x \rightarrow+\infty .
$$

Lemma 3 , with $k=3$, implies that

$$
\sum_{-\sqrt{x} \leqslant m \leqslant \sqrt{x}}\left(x-m^{2}\right)^{3 / 2}=\frac{3}{8} \pi x^{2}+O(x), x \rightarrow+\infty,
$$

and we get

$$
A_{4}(x)=\frac{\pi x^{2}}{2}+o(x \cdot \log \log x), x \rightarrow+\infty,
$$

in contradiction to (3). Thus (13) is impossible, and the proof of (2) is complete.

\section{Remarks}

1. The method used here is the derivation of a $o$-estimate for $R_{4}(x)$ from an assumed $o$-estimate for $R_{3}(x)$. Thus an improved $\Omega$-estimate for $R_{4}(x)$ would immediately give an improvement on $(2)$, by the same method.

2. This process can be applied to give an $O$-estimate for $R_{3}(x)$, given an $O$-estimate for $R_{2}(x)$. If we start with Vinogradov's result [2]

$$
R_{2}(x)=O\left(x^{\frac{17}{53}+\epsilon}\right), \epsilon>0, x \rightarrow+\infty,
$$

we get

$$
R_{3}(x)=O\left(x^{\frac{87}{106}+\epsilon}\right), \epsilon>0, x \rightarrow+\infty
$$

an estimate which is, however, weaker than (1).

\section{References}

[1] K. Chandrasekharan and R. Narashimhan. Hecke's functional equation and the average order of arithmetical functions. Acta Arithmetica 6, 487-503 (1961).

[2] I. M. Vinogradov. On the number of integral points in the interior of a circle (Russian), Bulletin Acad. Sci. Leningrad 7, 313-336 (1932).

[3] I. M. Vinogradov, On the number of integral points in a given domain (Russian), Izv Akad. Nauk SSSR Ser. Mat. 24, $777-786$ (1960).

[4] Arnold Walfisz, Gitterpunkte in mehrdimensionalen Kugeln, Warsaw, Panstwowe Wydawnictwo Naukowe (1957). 